COMMUNICATIONS IN

NUMBER THEORY AND PHYSICS

Volume 1, Number 2, 323-346, 2007

\title{
Fuchsian equations of type DN
}

\author{
Vasily Golyshev and Jan Stienstra
}

\begin{abstract}
We prove that a generic differential operator of type DN is irreducible, regular, (anti)self-adjoint, and has quasiunipotent local monodromies. We prove that the defining matrix of a DN operator can be recovered from the expression of the operator as a polynomial in $t$ and $\partial_{t}$.
\end{abstract}

\section{Introduction}

Let $A=\left(a_{i j}\right)_{0 \leqslant i, j \leqslant N}$ be a matrix with entries in $\mathbb{C}$ satisfying $a_{i j}=0$ if $i-j>1$ and $a_{i j}=1$ if $i-j=1$ and $a_{i j}=a_{N-j, N-i}$. With this matrix, we associate a differential operator $L_{A, \infty}$ as follows. Let $\tilde{A}=\left(a_{i j} \partial_{t}{ }^{j-i+1}\right)$ and $\partial_{t}=\frac{d}{d t}$. The right determinant $\operatorname{det}_{\text {right }}\left(t \partial_{t}-\tilde{A}\right)$ is a differential operator which is uniquely divisible from the right by $\partial_{t}$. We define

$$
L_{A, \infty}=\operatorname{det}_{\text {right }}\left(t \partial_{t}-\tilde{A}\right) \partial_{t}^{-1} .
$$

The notation $L_{A, \infty}$ signifies its dependence on $A$ and the fact that the space of its solutions has maximally unipotent monodromy at infinity. For $\Phi$ in a given module over the ring of the differential operators (e.g., that of functions of the variable $t$ ), the differential equation $L_{A, \infty} \Phi=0$ was called the determinantal equation of order $N$, or simply equation DN, in [5]. Theorem 5.8 in the present paper characterizes (through a constructive bijective correspondence) these operators as precisely those differential operators of the form

$$
\left(t \partial_{t}\right)^{N} t+\sum_{p=1}^{N+1} g_{p}\left(t \partial_{t}\right) \partial_{t}{ }^{p-1}
$$

with $g_{p}$ a polynomial of degree $\leqslant N-p+1$ in $t \partial_{t}$, such that $g_{p}$ (argument) $=$ $(-1)^{N-p+1} g_{p}(-$ argument $-p)$.

The construction of the differential operators of type DN in [5] is motivated by mirror symmetry for (complete intersections in) minimal Fano varieties. Recall that an algebraic variety $V$ over $\mathbb{C}$ is said to be a Fano variety if its anticanonical class $-K_{V}$ is ample. We say that a Fano variety $V$ of dimension $N$ is minimal if its cohomology is as small as it can be: 
$H^{2 k}(V, \mathbb{Z})=\mathbb{Z}$ and $H^{2 k+1}(V, \mathbb{Z})=0$ for all $0 \leq k \leq N$. Projective spaces are examples of minimal Fanos. The Hard Lefschetz theorem guarantees that a complete intersection in a minimal variety is almost minimal, in the sense that it only may acquire non-trivial cohomology in the middle dimension. Given a minimal Fano $V$, we introduce the upper triangular matrix of its normalized two-point Gromov-Witten invariants. Namely, the entry $a_{i j}$ of $A=A_{\mathrm{GW}(V)}$ is the GW invariant defined informally as

$(j-i+1) / \operatorname{deg} V \times$ the number of maps $\mathbb{P}^{1} \longrightarrow V$ of degree $j-i+1$ such that 0 goes to a point on a generic representative of the cohomology class $H^{j}$ and $\infty$ to a point on a generic representative of $H^{N-i}$.

The maps are considered up to automorphisms of $\mathbb{P}^{1}$ that fix 0 and $\infty$. The matrix $A_{\mathrm{GW}(V)}$ is 'symmetric': $a_{i j}=a_{N-j, N-i}$. We remark here that this construction was essentially introduced by Dubrovin under the name of 'second structural connection'. For a textbook description of Dubrovin's theory, we refer the reader to [9].

There is, however, no general characterization of the differential operators of type DN (or matrices $A$ ) that actually arise from the enumerative geometry of Fano varieties. What are the properties that distinguish $L_{A_{\mathrm{GW}}}$ 's within the affine space of all $L_{A}$ 's? A statement of the mirror conjecture asserts that $L_{A_{\mathrm{GW}}}$ are of Picard-Fuchs type. Namely, there exists a pencil of varieties $E_{t}$ over $\mathbb{A}^{1}$ (the so called Landau-Ginzburg model for $V$ ) and a relative differential form $\omega_{t}$ in this pencil such that the fibrewise period integrals of this form as multivalued functions on the base are annihilated by $L_{A_{\mathrm{GW}}}$ :

$$
\text { Mirror Conjecture }:\left\{L_{A_{\mathrm{GW}}}{ }^{\prime} \mathrm{s}\right\} \subset\left\{L_{\mathrm{PF}} \text { 's }\right\} \text {. }
$$

A natural question, in the light of mirror symmetry, is then to ask which of the above differential operators are Picard-Fuchs operators for one-parameter families of complex varieties, or more generally variations of Hodge structure.

According to Dubrovin (see [9, Chapter II]) a generic differential operator DN is associated with a Fuchsian connection, hence is regular. We prove that it is irreducible and coincides, up to a sign, with its adjoint. It is known to have maximal unipotent monodromy at $t=\infty$, and we show in this note that it has quasi-unipotent monodromies elsewhere. Regularity and quasiunipotence of local monodromies are definite requirements for Picard-Fuchs operators by Deligne, but do not suffice. 
Unfortunately, we know no general way to tell which of the differential operators of type DN do also satisfy further requirements for coming from a variation of $\mathbb{R}$-Hodge structures or being globally crystalline.

\section{Algebraic preliminaries}

Conventions 2.1. In this section, $R$ is an associative ring with unity. We do not want to use ugly looking indices such as $a_{i N}$. For this reason, we set $n=N$, and produce our DNs starting with a matrix of size $n+1$ whose row and column indices run from 0 to $n$.

Definition 2.2. The right determinant $\operatorname{det}_{\text {right }}(M)$ of a square matrix $M=$ $\left(M_{i j}\right)_{0 \leqslant i, j \leqslant n}$ with entries in $R$ is defined by expanding with respect to the right column:

$$
\operatorname{det}_{\text {right }}(M)=\sum_{i=0}^{n} M_{i n} C_{i n}
$$

where $C_{i n}$ is the cofactor of the element $M_{i n}$; the cofactor $C_{i n}$ is in turn a right determinant times a $\operatorname{sign}(-1)^{i+n}$.

By fully expanding this recursive definition one sees that

$$
\operatorname{det}_{\text {right }}(M)=\sum_{\sigma} \operatorname{sign}(\sigma) M_{\sigma(n), n} \cdots M_{\sigma(0), 0}
$$

where $\sigma$ runs over all permutations of $\{0, \ldots, n\}$. In particular, if $M$ has a row of 0 's, then $\operatorname{det}_{\text {right }}(M)=0$.

Proposition 2.3. If two matrices $M=\left(M_{i j}\right)_{0 \leqslant i, j \leqslant n}$ and $M^{\prime}=\left(M_{i j}^{\prime}\right)_{0 \leqslant i, j \leqslant n}$ are related by $M_{i j}^{\prime}=(-1)^{j-i+1} M_{i j}$, then $\operatorname{det}_{\text {right }}\left(M^{\prime}\right)=(-1)^{n+1} \operatorname{det}_{\text {right }}(M)$.

Definition 2.4. Let $M=\left(M_{i j}\right)$ be a square matrix with entries in $R$. We say that $M$ is almost triangular ${ }^{1}$ if $M_{i j}=0$ for $i>j+1$, and $M_{j+1, j}=-1$.

For an almost triangular matrix $M$ one can reformulate Definition 2.2 as a simple inductive algorithm (cf. [3]) that consecutively expresses a principal

\footnotetext{
${ }^{1}$ In numerical linear algebra, a matrix $M$ is said to be "upper (resp. lower) almost triangular" or in "upper (resp. lower) Hessenberg form" if $M_{i j}=0$ for $i>j+1$ (resp. $j>i+1$ ). Our almost triangular matrices are thus the "upper almost triangular" matrices of numerical linear algebra subject to the additional requirement that all the subdiagonal elements be $-1 \mathrm{~s}$.
} 
minor in terms of preceding ones:

$$
P_{0}=1, \quad P_{j+1}=\sum_{i=0}^{j} M_{i j} P_{i}, \operatorname{det}_{\text {right }}(M)=P_{n+1} .
$$

More generally, $P_{0}, \ldots, P_{n+1}$ are the right principal minors of $M$.

Proposition 2.5. Let $M$ be an almost triangular matrix of size n. Define the elements $Q_{j}$ in $R$ inductively by:

$$
Q_{0}=1, \quad Q_{j+1}=\sum_{i=0}^{j} Q_{i} M_{n-j, n-i} .
$$

Then $Q_{n+1}=\operatorname{det}_{\text {right }}(M)$.

Proof. When fully expanded both inductive algorithms lead to:

$$
P_{n+1}=Q_{n+1}=\sum M_{\left(i_{k}, i_{k+1}-1\right)} M_{\left(i_{k-1}, i_{k}-1\right)} \cdots M_{\left(i_{0}, i_{1}-1\right)}
$$

where the sum is over all sequences $\left(i_{0}, \ldots, i_{k+1}\right)$ of integers satisfying $0=$ $i_{0}<i_{1}<\cdots<i_{k}<i_{k+1}=n+1$.

Definition 2.6. For a square matrix $M=\left(M_{i j}\right)_{0 \leqslant i, j \leqslant n}$ with entries in $R$ let $M^{\tau}$ be the matrix with $(i, j)$-entry $M_{i j}^{\tau}=M_{n-j, n-i}$.

Thus $M^{\tau}$ is the "transpose of $M$ with respect to the anti-diagonal". It relates to the ordinary transpose $M^{t}$ as $M^{\tau}=\mathrm{J} M^{t} \mathrm{~J}$, where $\mathrm{J}=\left(\mathrm{J}_{i j}\right)_{0 \leqslant i, j \leqslant n}$ denotes the matrix with $\mathrm{J}_{i j}=1$ if $i+j=n$ and $\mathrm{J}_{i j}=0$ otherwise.

Proposition 2.7. Let $R$ be as above and let $E$ be a right $R$-module. Let $\xi_{0}, \ldots, \xi_{n}$ be elements of $E$. Let $M=\left(M_{i j}\right)_{0 \leqslant i, j \leqslant n}$ be an almost triangular matrix over $R$. Then

$$
\left(\xi_{0}, \ldots, \xi_{n}\right) M^{\tau}=(0, \ldots, 0) \Longrightarrow \xi_{0} \operatorname{det}_{\text {right }}(M)=0 .
$$

Proof. If $\left(\xi_{0}, \ldots, \xi_{n}\right) M^{\tau}=(0, \ldots, 0)$, then $\xi_{j+1}=\sum_{i=0}^{j} \xi_{i} M_{n-j, n-i}$ for $j=$ $0, \ldots, n-1$. This implies $\xi_{j}=\xi_{0} Q_{j}$ for $0 \leqslant j \leqslant n$ with $Q_{j}$ as in Proposition 2.5. Now $\left(\xi_{0}, \ldots, \xi_{n}\right)\left(\right.$ right column of $\left.M^{\tau}\right)=0 \operatorname{implies}_{0} \xi_{0} \operatorname{det}_{\text {right }}(M)=0$.

Suppose that the ring $R$ is equipped an anti-involution ${ }^{\vee}$; i.e., a map $R \rightarrow$ $R, x \mapsto x^{\vee}$, such that $(x+y)^{\vee}=x^{\vee}+y^{\vee}$ and $(x y)^{\vee}=y^{\vee} x^{\vee}$ for all $x, y \in R$. 
Let $M=\left(M_{i j}\right)$ be an almost triangular matrix over $R$. Applying the antiinvolution to the inductive algorithm in Proposition 2.5 we obtain

$$
Q_{0}^{\vee}=1, \quad Q_{j+1}^{\vee}=\sum_{i=0}^{j} M_{n-j, n-i}^{\vee} Q_{i}^{\vee},
$$

which is in fact the inductive algorithm in the paragraph below Definition 1.4 for computing the right determinant of the matrix $M^{\tau \vee}$ (on matrices ${ }^{\vee}$ acts componentwise). Thus

Proposition 2.8. One has $\left(\operatorname{det}_{\text {right }}(M)\right)^{\vee}=\operatorname{det}_{\text {right }}\left(M^{\tau \vee}\right)$.

\section{Almost triangular matrices over the Weyl algebra}

Let $B=\mathbb{Q}\left[a_{i j}\right]$ be the commutative polynomial ring in the variables $a_{i j}$ with $0 \leqslant i \leqslant j \leqslant n$. We put a grading on $B$ so that $a_{i j}$ is homogeneous of degree $j-i+1$. Let $R$ be the Weyl algebra over $B$, i.e., the non-commutative polynomial ring $B\left[u, u^{*}\right]$ with centre $B$ modulo the commutation relation $u u^{*}-u^{*} u=1$. We define the matrix $\tilde{A}=\left(\tilde{A}_{i j}\right)_{0 \leqslant i, j \leqslant n}$ with entries in $R$ by $\tilde{A}_{i j}=0$ if $i>j+1, \tilde{A}_{i j}=1$ if $i=j+1$ and

$$
\tilde{A}_{i j}=a_{i j} u^{j-i+1} \quad \text { if } \quad i<j+1 .
$$

Then $u u^{*}-\tilde{A}$ is an almost triangular matrix; here, and henceforth, we simplify the notation by writing just $u u^{*}$ instead of $u u^{*}$ times the identity matrix of size $n+1$. Using the inductive algorithm in the paragraph below Definition 2.4 one checks that its right determinant has an expansion of the form:

$$
\operatorname{det}_{\text {right }}\left(u u^{*}-\tilde{A}\right)=\left(u u^{*}\right)^{n+1}+\sum_{p=1}^{n+1} \sum_{k=0}^{n-p+1} x_{k}^{(p)} u^{p}\left(u u^{*}\right)^{k}
$$

in which $x_{k}^{(p)}$ is a homogeneous element of degree $p$ in $B$.

Define, for $p \geqslant 1$, the endomorphisms $\tau^{\geqslant p}$ and $\tau^{\leqslant p}$ of the ring $B$ by

$$
\begin{aligned}
& \tau^{\geqslant p}\left(a_{i j}\right)=0 \quad \text { if } \quad j-i+1<p, \quad \tau^{\geqslant p}\left(a_{i j}\right)=a_{i j} \quad \text { if } \quad j-i+1 \geqslant p, \\
& \tau^{\leqslant p}\left(a_{i j}\right)=0 \quad \text { if } \quad j-i+1>p, \quad \tau^{\leqslant p}\left(a_{i j}\right)=a_{i j} \quad \text { if } \quad j-i+1 \leqslant p .
\end{aligned}
$$

Then $\tau^{\leqslant p}\left(x_{k}^{(p)}\right)=x_{k}^{(p)}$ and $\tau^{\geqslant p}\left(x_{k}^{(p)}\right)$ is a linear combination of the variables $a_{i, p+i-1}$, since $x_{k}^{(p)}$ is homogeneous of degree $p$. Thus for every $p, 1 \leqslant p \leqslant n$, 
there is a square matrix $K^{(p)}=\left(K_{k i}^{(p)}\right)$ over $\mathbb{Q}$ of size $n+2-p$ such that

$$
\tau^{\geqslant p}\left(x_{k}^{(p)}\right)=\sum_{i=0}^{n-p+1} K_{k i}^{(p)} a_{i, p+i-1} .
$$

Lemma 3.1. The matrix $K^{(p)}$ is invertible over $\mathbb{Q}$, for every $p \geqslant 1$.

Proof. For $j \geqslant i \geqslant 0$, let $E^{(i, j)}=\left(E_{k l}^{(i, j)}\right)_{0 \leqslant k, l \leqslant n}$ denote the matrix with $E_{i j}^{(i, j)}=u^{j-i+1}, E_{k+1, k}^{(i, j)}=1$ for $0 \leqslant k \leqslant n-1$ and $E_{k l}^{(i, j)}=0$ else. The recursion rule in the paragraph below Definition 2.4 yields

$$
\begin{aligned}
\operatorname{det}_{\text {right }}\left(u u^{*}-E^{(i, p+i-1)}\right)-\left(u u^{*}\right)^{n+1} & =-\left(u u^{*}\right)^{n+1-p-i} u^{p}\left(u u^{*}\right)^{i} \\
=-u^{p}\left(u u^{*}-p\right)^{n+1-p-i}\left(u u^{*}\right)^{i} & =\sum_{k=0}^{n-p+1} K_{k i}^{(p)} u^{p}\left(u u^{*}\right)^{k}
\end{aligned}
$$

Invertibility of the matrix $K^{(p)}$ therefore follows from the fact that the elements $\left(u u^{*}-p\right)^{n+1-p-i}\left(u u^{*}\right)^{i}$ with $0 \leqslant i \leqslant n-p+1$ are linearly independent.

Theorem 3.2. Consider the polynomial ring $\Lambda=\mathbb{Q}\left[\lambda_{k}^{(p)}\right]_{0 \leqslant k \leqslant n-p+1 \leqslant n}$ together with the ring morphism $\varphi: \Lambda \rightarrow B, \varphi\left(\lambda_{k}^{(p)}\right)=x_{k}^{(p)}$. Then $\varphi$ is an isomorphism.

Proof. From the paragraph after Equation (3.1) and Lemma 3.1 we see that $a_{i, p+i-1}$ is a linear combination of the elements $x_{k}^{(p)}$ with $0 \leqslant k \leqslant n-p+1$ plus a polynomial in the elements $a_{i j}$ with $j-i+1<p$. So, by induction, $a_{i, p+i-1}$ is in the image of $\varphi$. Hence $\varphi$ is surjective. Put a grading on $\Lambda$ by declaring that $\lambda_{k}^{(p)}$ is homogeneous of degree $p$. Then $\varphi$ is a morphism between graded $\mathbb{Q}$-algebras. Moreover, for every $p$, the homogeneous pieces of degree $p$ in $\Lambda$ and $B$ are $\mathbb{Q}$-vector spaces of the same dimension. So in each degree $\varphi$ is a surjective linear map between vector spaces of the same dimension. Hence $\varphi$ is an isomorphism.

In down to earth terms the theorem means:

Corollary 3.3. The matrix $A$ can be reconstructed from the expansion of $\operatorname{det}_{\text {right }}\left(u u^{*}-\tilde{A}\right)$.

Proof. By the theorem, the coefficients $x_{k}^{(p)}$ in the expansion of $\operatorname{det}_{\text {right }}\left(u u^{*}-\right.$ $\tilde{A})$ are algebraically independent and the matrix entries $a_{i j}$ are polynomials 
in the $x_{k}^{(p)}$ 's. This remains true when the $x_{k}^{(p)}$ 's are specialized to complex numbers, leading to an almost triangular matrix $-A$ over $\mathbb{C}$.

\section{Realizations}

In this section, $R$ is the Weyl algebra over $\mathbb{C}$, i.e., the non-commutative polynomial ring $\mathbb{C}\left[u, u^{*}\right]$ with centre $\mathbb{C}$ modulo the relation $u u^{*}-u^{*} u=1$. Further, $A=\left(a_{i j}\right)_{0 \leqslant i, j \leqslant n}$ is a matrix with entries in $\mathbb{C}$ such that $-A$ is almost triangular. We define the matrix $\tilde{A}=\left(\tilde{A}_{i j}\right)_{0 \leqslant i, j \leqslant n}$ with entries in $R$ by

$$
\tilde{A}_{i j}=a_{i j} u^{j-i+1} \text {. }
$$

We want to apply the result of Proposition 2.7 to the almost triangular matrix $u u^{*}-\tilde{A}^{\tau}$. So, we need a right $R$-module $E$ and in it elements $\xi_{0}, \ldots, \xi_{n}$ such that

$$
\left(\xi_{0}, \ldots, \xi_{n}\right)\left(u u^{*}-\tilde{A}\right)=(0, \ldots, 0) .
$$

We will present two realizations of this situation, called the first-model and the second-model. The terminology first-model and second-model refers to the fact that these give the first and the second structure connection, respectively, in [9, Chapter II]; see 4.2 and 4.3 below. For both realizations, we use an isomorphism of the Weyl algebra with an algebra of differential operators:

$$
\begin{array}{ccccc}
\text { first-model: } & R \stackrel{\sim}{\rightarrow} \mathbb{C}\left[z, \partial_{z}\right], & u \mapsto z, & u^{*} \mapsto-\partial_{z} & \left(\partial_{z}=\frac{d}{d z}\right), \\
\text { second-model: } & R \stackrel{\sim}{\rightarrow} \mathbb{C}\left[t, \partial_{t}\right], & u \mapsto \partial_{t}, & u^{*} \mapsto t & \left(\partial_{t}=\frac{d}{d t}\right) .
\end{array}
$$

These isomorphisms with the Weyl algebra yield the isomorphism

$$
\mathbb{C}\left[t, \partial_{t}\right] \stackrel{\sim}{\rightarrow} \mathbb{C}\left[z, \partial_{z}\right], \quad t \mapsto-\partial_{z}, \quad \partial_{t} \mapsto z .
$$

This means that the first-model as a left $\mathbb{C}\left[z, \partial_{z}\right]$-module is the Fourier transform of the second-model as a left $\mathbb{C}\left[t, \partial_{t}\right]$-module in the standard sense (cf. [8, p. 71]).

The algebra $\mathbb{C}\left[z, \partial_{z}\right]$ admits an anti-involution ${ }^{\vee}$ which is the identity on $\mathbb{C}$ and satisfies

$$
z^{\vee}=z, \quad \partial_{z}^{\vee}=-\partial_{z}
$$

Using this anti-involution, one can turn a right module $E$ over $\mathbb{C}\left[z, \partial_{z}\right]$ into a left module by defining

$$
a \xi=\xi a^{\vee} \quad \text { for } \quad \xi \in E, a \in \mathbb{C}\left[z, \partial_{z}\right] .
$$


The same applies, of course, to $\mathbb{C}\left[t, \partial_{t}\right]$.

\subsection{First-model}

For the first-model we take the free module $E$ with basis $\xi_{0}, \ldots, \xi_{n}$ over the ring of Laurent polynomials $\mathbb{C}\left[z, z^{-1}\right]$ and give it the structure of a right module over $\mathbb{C}\left[z, \partial_{z}\right]$ by defining

$$
\left(\xi_{0}, \ldots, \xi_{n}\right) \partial_{z}=\left(\xi_{0}, \ldots, \xi_{n}\right)\left(\mathrm{I}-\tilde{A}_{1}\right) z^{-1}
$$

where I is the identity matrix of size $n+1$ and $\tilde{A}_{1}=\left(a_{i j} z^{j-i+1}\right)$.

This definition implies

$$
\left(\xi_{0}, \ldots, \xi_{n}\right)\left(-z \partial_{z}-\tilde{A}_{1}\right)=\left(\xi_{0}, \ldots, \xi_{n}\right)\left(-\partial_{z} z+\left(\mathrm{I}-\tilde{A}_{1}\right)\right)=(0, \ldots, 0) .
$$

So, Proposition 2.7 implies

$$
\xi_{0} \operatorname{det}_{\text {right }}\left(-z \partial_{z}-\tilde{A}_{1}^{\tau}\right)=0 .
$$

Passing from right to left modules with the involution $\vee$ and also using Proposition 2.8 we can rewrite the above formulas as

First-model connection: $\left(\partial_{z} \xi_{0}, \ldots, \partial_{z} \xi_{n}\right)=\left(\xi_{0}, \ldots, \xi_{n}\right)\left(\tilde{A}_{1}-\mathrm{I}\right) z^{-1}$,

First-model differential equation: $\operatorname{det}_{\text {right }}\left(\partial_{z} z-\tilde{A}_{1}\right) \xi_{0}=0$.

\subsection{First-model (bis)}

We introduce the diagonal matrix

$$
\mathrm{\top}=\operatorname{diag}(0,1, \ldots, n) .
$$

Then $z^{\top}=\operatorname{diag}\left(1, z \ldots, z^{n}\right)$ and $\tilde{A}_{1}=z^{-\top} A z^{\top} z$. Thus, by the change of coordinates $\left(\zeta_{0}, \ldots, \zeta_{n}\right)=\left(\xi_{0}, \ldots, \xi_{n}\right) z^{-\mathrm{T}}$ one can put the above $1^{\text {st }}$-model connection in the format of $[9$, p. 53 , formula (1.23)]:

$$
\left(\partial_{z} \zeta_{0}, \ldots, \partial_{z} \zeta_{n}\right)=\left(\zeta_{0}, \ldots, \zeta_{n}\right)\left(A-(\mathrm{I}+\mathrm{T}) z^{-1}\right)
$$

\subsection{Second-model}

For the second-model, we take the free module $E^{\prime}$ with basis $\eta_{0}, \ldots, \eta_{n}$ over the ring $\mathbb{C}\left[t, \chi_{A}^{-1}\right]$, where $\chi_{A}=\operatorname{det}(A-t)$ is the characteristic polynomial 
of the matrix $A=\left(a_{i j}\right)$. We give $E^{\prime}$ the structure of a right module over $\mathbb{C}\left[t, \partial_{t}\right]$ by defining

$$
\left(\eta_{0}, \ldots, \eta_{n}\right) \partial_{t}=\left(\eta_{0}, \ldots, \eta_{n}\right) \mathrm{T}(A-t)^{-1}
$$

Let $\tilde{A}=\left(a_{i j} \partial_{t}{ }^{j-i+1}\right)$ and $V=\operatorname{diag}\left(1, \partial_{t}, \partial_{t}{ }^{2}, \ldots, \partial_{t}{ }^{n}\right)$. Then $V \tilde{A}=\partial_{t} A V$ and $V \partial_{t} t=\left(\partial_{t} t+\mathrm{T}\right) V$ and

$$
\left(\eta_{0}, \ldots, \eta_{n}\right)\left(V \tilde{A}-V \partial_{t} t\right)=\left(\eta_{0}, \ldots, \eta_{n}\right)\left(\partial_{t}(A-t)-\mathrm{T}\right) V=(0, \ldots, 0) .
$$

Thus, if we define the elements $\xi_{0}^{\prime}, \ldots, \xi_{n}^{\prime}$ in $E^{\prime}$ by $\xi_{k}^{\prime}=\eta_{k}\left(\partial_{t}\right)^{k}$, then

$$
\left(\xi_{0}^{\prime}, \ldots, \xi_{n}^{\prime}\right)\left(\partial_{t} t-\tilde{A}\right)=(0, \ldots, 0)
$$

So, Proposition 2.7 implies

$$
\xi_{0}^{\prime} \operatorname{det}_{\text {right }}\left(\partial_{t} t-\tilde{A}^{\tau}\right)=0
$$

Passing from right to left modules with the involution ${ }^{\vee}$ and also using 2.8 we can write this formula also as

$$
\operatorname{det}_{\text {right }}\left(-t \partial_{t}-\tilde{A}^{\vee}\right) \eta_{0}=0
$$

Note $\tilde{A}^{\vee}=\left(a_{i j}\left(-\partial_{t}\right)^{j-i+1}\right)$. Proposition 2.3 enables one to rewrite the above formulas as

Second-model connection: $\quad\left(\partial_{t} \eta_{0}, \ldots, \partial_{t} \eta_{n}\right)=\left(\eta_{0}, \ldots, \eta_{n}\right) \mathrm{T}(t-A)^{-1}$,

Second-model differential equation: $\operatorname{det}_{\text {right }}\left(t \partial_{t}-\tilde{A}\right) \eta_{0}=0$.

This second-model connection has exactly the form of $[9$, p. 53, formula (1.22)].

Remark 4.1. Note that the top row of $T$ is zero and that therefore $\eta_{0}$ is actually absent from the right hand side of the second-model connection formula. Consequently, the $\mathbb{C}\left[t, \chi_{A}^{-1}\right]$-submodule $E^{\prime \prime}$ of $E^{\prime}$ with basis $\eta_{1}, \ldots, \eta_{n}$ is stable under the action of $\partial_{t}$, i.e., $\partial_{t} E^{\prime \prime} \subset E^{\prime \prime}$. Moreover, $\partial_{t} \eta_{0} \in E^{\prime \prime}$ and this implies that the class of $\eta_{0}$ modulo $E^{\prime \prime}$ is horizontal for the connection on the quotient $E^{\prime} / E^{\prime \prime}$. The counterpart of this structure is that the secondmodel differential operator is divisible on the right by $\partial_{t}$; see Lemma 5.1. 


\subsection{Connection versus differential equation}

We remind the reader how the problem of finding horizontal sections for a connection relates to that of solving a differential equation.

Let $\mathcal{A}$ be a commutative algebra with a left action of $\mathbb{C}\left[t, \partial_{t}\right]$ that satisfies the Leibniz rule. Assume also that $\mathcal{A}$ acts on the right on the free $\mathcal{A}$-module $E \otimes_{\mathbb{C}\left[t, \chi_{A}^{-1}\right]} \mathcal{A}$ with the Leibniz rule

$$
\partial_{t}(e a)=\left(\partial_{t} e\right) a+e\left(\partial_{t} a\right), \quad e \in E \otimes_{\mathbb{C}\left[t, \chi_{A}^{-1}\right]} \mathcal{A}, \quad a \in \mathcal{A}
$$

Let $\Phi$ be an invertible matrix of size $n+1$ whose entries are elements of $\mathcal{A}$, and assume that

$$
\partial_{t}\left(\left(\eta_{0}, \ldots, \eta_{n}\right) \Phi\right)=(0, \ldots, 0)
$$

(In practice, $\mathcal{A}$ would of course be the algebra of analytic functions on some open subset $U$ of $\mathbb{C} ;\left(\eta_{0}, \ldots, \eta_{n}\right) \Phi$ is then a basis for the space of horizontal analytic sections over $U$ for the connection.)

From

$$
\begin{aligned}
\partial_{t}\left(\left(\eta_{0}, \ldots, \eta_{n}\right) \Phi\right) & =\left(\partial_{t}\left(\eta_{0}, \ldots, \eta_{n}\right)\right) \Phi+\left(\eta_{0}, \ldots, \eta_{n}\right) \partial_{t} \Phi \\
& =\left(\eta_{0}, \ldots, \eta_{n}\right)\left(\mathrm{T}(t-A)^{-1} \Phi+\partial_{t} \Phi\right)=(0, \ldots, 0)
\end{aligned}
$$

we see that $\Phi$ is "a fundamental solution matrix" of the system

$$
\partial_{t} \Phi=\mathrm{T}(A-t)^{-1} \Phi
$$

The trivial fact $\partial_{t}\left(\Phi^{-1} \Phi\right)=0$ implies $\partial_{t} \Phi^{-1}=\Phi^{-1} \mathrm{~T}(t-A)^{-1}$. One can therefore apply the arguments in section 4.3 to the columns of $\Phi^{-1}$ in place of $\left(\eta_{0}, \ldots, \eta_{n}\right)$. Thus, the left-most column of $\Phi^{-1}$ is componentwise annihilated by the differential operator $\operatorname{det}_{\text {right }}\left(t \partial_{t}-\tilde{A}\right)$. So the elements appearing as entries in the left-most column of $\Phi^{-1}$ are solutions of the differential equation $\operatorname{det}_{\text {right }}\left(t \partial_{t}-\tilde{A}\right) f=0$.

\section{Differential operators of type $D N$}

Lemma 5.1. Let $A=\left(a_{i j}\right)_{0 \leqslant i, j \leqslant n}$ be a matrix with entries in $\mathbb{C}$ such that $-A$ is almost triangular. Let $\tilde{A}$ denote the matrix with $(i, j)$-entry $a_{i j} \partial_{t}{ }^{j-i+1}$. Then the differential operator $\operatorname{det}_{\text {right }}\left(t \partial_{t}-\tilde{A}\right)$ in the second-model differential equation is uniquely divisible from the right by $\partial_{t}$. 
Proof. This follows from Proposition 2.5 and the observation that in the matrix $t \partial_{t}-\tilde{A}$ the entry in position $(i, j)$ is uniquely divisible from the right by $\partial_{t}$ if $i \leqslant j$.

Definition 5.2. With $A$ and $\tilde{A}$ as in Lemma 5.1 we define the differential operator

$$
L_{A, \infty}=\operatorname{det}_{\text {right }}\left(t \partial_{t}-\tilde{A}\right) \partial_{t}^{-1} .
$$

Proposition 5.3. The operators $L_{A, \infty}$ one obtains with Definition 5.2 are precisely the operators of the form

$$
\left(t \partial_{t}\right)^{n} t+\sum_{p=1}^{n+1} g_{p}\left(t \partial_{t}\right) \partial_{t}^{p-1}
$$

with $g_{p}$ a polynomial of degree $\leqslant n-p+1$ in $t \partial_{t}$.

Proof. Setting $u=\partial_{t}$ and $u^{*}=t$ in the right-hand side of (3.1) and applying the anti-involution $\vee$ gives

$$
\begin{aligned}
L_{A, \infty} & =(-1)^{n}\left(t\left(\partial_{t} t\right)^{n}+\sum_{p=1}^{n+1} \sum_{k=0}^{n-p+1} x_{k}^{(p)} \partial_{t}^{p-1}\left(\partial_{t} t\right)^{k}\right)^{\vee} \\
& =\left(t \partial_{t}\right)^{n} t+\sum_{p=1}^{n+1} \sum_{k=0}^{n-p+1}(-1)^{n+k+p-1} x_{k}^{(p)}\left(t \partial_{t}\right)^{k} \partial_{t}^{p-1}
\end{aligned}
$$

Continuing the discussion in section 4.4 we see that the entries in the leftmost column of the matrix $\partial_{t} \Phi^{-1}$ are solutions to the differential equation $L_{A, \infty} g=0$.

Let us now consider the ring of differential operators $\mathbb{C}\left[w, w^{-1}, \partial_{w}\right]$ on the torus $\operatorname{Spec} \mathbb{C}\left[w, w^{-1}\right]$. The substitution $t=w^{-1}$ transforms $\partial_{t}$ into $-w^{2} \partial_{w}$ and $t \partial_{t}$ into $-w \partial_{w}$. Thus the differential operator $L_{A, \infty}$, expanded as in Proposition 5.3, transforms into

$$
\left(-w \partial_{w}\right)^{n} w^{-1}+\sum_{p=1}^{n+1} g_{p}\left(-w \partial_{w}\right)\left(-w^{2} \partial_{w}\right)^{p-1}
$$


Definition 5.4. With the notations as in the above paragraph we define the differential operator

$$
L_{A, 0}=\left(w \partial_{w}\right)^{n}+\sum_{p=1}^{n+1}(-1)^{n} g_{p}\left(-w \partial_{w}\right)\left(-w^{2} \partial_{w}\right)^{p-1} w
$$

This means, according to the above paragraph, that the substitution $t=w^{-1}$ transforms $L_{A, \infty}$ into $(-1)^{n} L_{A, 0} w^{-1}$.

Proposition 5.5. The operators $L_{A, 0}$ one obtains with Definition 5.4 are precisely the operators of the form

$$
\left(w \partial_{w}\right)^{n}+\sum_{p=1}^{n+1} w^{p} G_{p}\left(w \partial_{w}\right) \prod_{l=1}^{p-1}\left(w \partial_{w}+l\right)
$$

where $G_{p}$ is a polynomial of degree $\leqslant n+1-p$ in $w \partial_{w}$, related to the polynomial $g_{p}$ from Proposition 5.3 by:

$$
G_{p}(\text { argument })=(-1)^{n-p+1} g_{p}(-\operatorname{argument}-p) \text {. }
$$

Proof. Note $g_{p}\left(-w \partial_{w}\right)\left(w^{2} \partial_{w}\right)^{p-1} w=w^{p} g_{p}\left(-w \partial_{w}-p\right) \prod_{l=1}^{p-1}\left(w \partial_{w}+l\right)$.

The anti-involution $\vee$ on $\mathbb{C}\left[t, \partial_{t}\right]$ defined by $t^{\vee}=t, \partial_{t} \vee=-\partial_{t}$ maps a differential operator $L=\sum_{i, j} c_{i j} t^{i} \partial_{t}{ }^{j}$ to $L^{\vee}=\sum_{i, j} c_{i j}\left(-\partial_{t}\right)^{j} t^{i}$. Thus, $L^{\vee}$ is the adjoint of $L$ in the sense of [8, p. 55]. We want to determine the adjoint of the operator $L_{A, \infty}$. Recall from Definition 5.2 that

$$
L_{A, \infty}=\operatorname{det}_{\text {right }}\left(t \partial_{t}-\tilde{A}\right) \partial_{t}^{-1}
$$

where $\tilde{A}$ is the matrix with $(i, j)$-entry $a_{i j} \partial_{t}{ }^{j-i+1}$. Using Proposition 2.8 we find:

$$
L_{A, \infty}^{\vee}=-\partial_{t}^{-1} \operatorname{det}_{\text {right }}\left(-\partial_{t} t-\tilde{A}^{\tau \vee}\right),
$$

where $\tilde{A}^{\tau}$ is the matrix with $(i, j)$-entry $a_{n-j, n-i} \partial_{t}{ }^{j-i+1}$.

Using Proposition 2.3, one sees

$$
L_{A, \infty}^{\vee}=(-1)^{n} \operatorname{det}_{\text {right }}\left(t \partial_{t}-\tilde{A}^{\tau}\right) \partial_{t}^{-1}=(-1)^{n} L_{A^{\tau}, \infty}
$$

Definition 5.4 now gives

$$
L_{A, 0}^{\vee}=(-1)^{n} w L_{A^{\tau}, 0} w^{-1}
$$


Theorem 5.6. The following three statements are equivalent

(i) $A=A^{\tau}$,

(ii) $L_{A, \infty}^{\vee}=(-1)^{n} L_{A, \infty}$,

(iii) $L_{A, 0}^{\vee}=(-1)^{n} w L_{A, 0} w^{-1}$.

Proof. This follows directly from the preceding paragraph and Corollory 2.3.

Definition 5.7. Following $[5,2.10]$ we call $L_{A, 0}$ a differential operator of type $\mathrm{DN}_{0,0}$ if the matrix $A$ satisfies the condition $A=A^{\tau}$. Under the same symmetry condition $L_{A, \infty}$ is called a differential operator of type $\mathrm{DN}_{\infty, 1}$. Here, $N=n$ is the order of the operator. Thus, for $n=3$ the operator $L_{A, \infty}$ is of type $D 3_{\infty, 1}$.

Theorem 5.8. (i) An operator $L$ of type $\mathrm{DN}_{\infty, 1}$ satisfies $L^{\vee}=(-1)^{n} L$.

(ii) The operators of type $\mathrm{DN}_{\infty, 1}$ are precisely the differential operators of the form

$$
\left(t \partial_{t}\right)^{n} t+\sum_{p=1}^{n+1} g_{p}\left(t \partial_{t}\right) \partial_{t}^{p-1}
$$

with $g_{p}$ a polynomial of degree $\leqslant n-p+1$, that satisfies

$$
g_{p}(\text { argument })=(-1)^{n-p+1} g_{p}(-\operatorname{argument}-p)
$$

(iii) An operator $L$ of type $\mathrm{DN}_{0,0}$ satisfies $\quad L^{\vee}=(-1)^{n} w L w^{-1}$.

(iv) The operators of type $\mathrm{DN}_{0,0}$ are precisely the differential operators of the form

$$
\left(w \partial_{w}\right)^{n}+\sum_{p=1}^{n+1} w^{p} G_{p}\left(w \partial_{w}\right) \prod_{l=1}^{p-1}\left(w \partial_{w}+l\right)
$$

where $G_{p}$ is a polynomial of degree $\leqslant n-p+1$, that satisfies

$$
G_{p}(\text { argument })=(-1)^{n-p+1} G_{p}(-\operatorname{argument}-p) .
$$


Proof. (i) follows from Theorem 5.6 and Definition 5.7.

(ii) Write the operator $L$ as in Proposition 5.3. Then $L^{\vee}=(-1)^{n} L$ becomes

$$
t\left(-\partial_{t} t\right)^{n}+\sum_{p=1}^{n+1}\left(-\partial_{t}\right)^{p-1} g_{p}\left(-\partial_{t} t\right)=(-1)^{n}\left[\left(t \partial_{t}\right)^{n} t+\sum_{p=1}^{n+1} g_{p}\left(t \partial_{t}\right) \partial_{t}^{p-1}\right]
$$

and boils down to

$$
g_{p}\left(t \partial_{t}\right) \partial_{t}^{p-1}=(-1)^{n+p-1} \partial_{t}^{p-1} g_{p}\left(-\partial_{t} t\right)=(-1)^{n+p-1} g_{p}\left(-t \partial_{t}-p\right) \partial_{t}^{p-1}
$$

for every $p \geqslant 1$. This proves (ii).

(iii) and (iv) follow directly from Theorem 5.6, (ii) and Proposition 5.5.

Example 5.9. For $\lambda \in \mathbb{C}$ consider the matrix

$$
A=\left(\begin{array}{ccc}
\lambda & -\frac{3}{2} \lambda^{2} & -\lambda^{3} \\
1 & -2 \lambda & -\frac{3}{2} \lambda^{2} \\
0 & 1 & \lambda
\end{array}\right)
$$

A straightforward calculation shows that for this matrix

$$
L_{A, \infty}=t^{3} \partial_{t}^{2}+3 t^{2} \partial_{t}+t-\lambda
$$

Divided by $t$, it yields a monic polynomial in $t \partial_{t}$ with coefficients in $\mathbb{C}(t)$. In the case $\lambda \neq 0$, one of these coefficients, $\lambda / t$, is not analytic at 0 hence, by the Fuchs criterion, the operator $L_{A, \infty}$ has an irregular singularity at $t=0$.

Remark 5.10. The above example shows that operators of type $\mathrm{DN}_{\infty, 1}$ may have irregular singularities. On the other hand, we will see in the next section that if the matrix $A$ is diagonalizable the corresponding operators $\mathrm{DN}_{\infty, 1}$ and $\mathrm{DN}_{0,0}$ have only regular singularities.

\section{Monodromy}

Assumption 6.1. In this section, we assume that $-A$ is almost triangular, $A$ is diagonalizable and $A=A^{\tau}$. 
We are going to investigate the local monodromies of the second-model connection,

$$
\left(\partial_{t} \eta_{0}, \ldots, \partial_{t} \eta_{n}\right)=\left(\eta_{0}, \ldots, \eta_{n}\right) \mathrm{T}(t-A)^{-1}
$$

or equivalently (cf. Section 4.4) of the system

$$
\partial_{t} \Phi=\mathrm{\top}(A-t)^{-1} \Phi
$$

It is clear that the singularities are at $\infty$ and at the eigenvalues of $A$. Since $-A$ is almost triangular, the last coordinate of every eigenvector of $A$ is non-zero (if it were zero, then the value of the pairing of the bottom row of $A$ with the eigenvector would be zero, hence so would be the $(n-1)$ th coordinate, and so forth). This implies that all eigenspaces of $A$ have dimension 1. Since $A$ is diagonalizable, this means that all eigenvalues of $A$ have multiplicity 1 . Let

$$
A=C \Lambda C^{-1}, \quad \Lambda=\operatorname{diag}\left(\lambda_{0}, \ldots, \lambda_{n}\right)
$$

By Assumption 6.1 and Definition 2.6, we have $A=A^{\tau}=\mathrm{J} A^{t} \mathrm{~J}$. Hence, $C \Lambda C^{-1}=\left(C^{t} \mathrm{~J}\right)^{-1} \Lambda C^{t} \mathrm{~J}$. Since $\lambda_{i} \neq \lambda_{j}$ if $i \neq j$, this implies that $C^{t} \mathrm{~J} C$ is a diagonal matrix. By multiplying $C$ on the right by a suitable diagonal matrix, we may assume

$$
C^{t} \mathrm{~J} C=\mathrm{I}
$$

Let $\mathbf{u}_{0}, \ldots, \mathbf{u}_{n}$ be the columns of the matrix $C$. So, $\mathbf{u}_{i}$ is an eigenvector of $A$ for the eigenvalue $\lambda_{i}$.

Theorem 6.2. With the above assumptions and notations

(i) the second-model system can be written as

$$
\partial_{t} \Phi=\sum_{j=0}^{n} \frac{1}{t-\lambda_{j}} S_{j} \Phi
$$

where $S_{j}=-\mathrm{T} C E_{j} C^{-1}$ and $E_{j}$ is the $(n+1) \times(n+1)$-matrix with 1 in position $(j, j)$ and zeros elsewhere;

(ii) the vectors $\mathbf{u}_{i}$ with $i \neq j$ are eigenvectors of $S_{j}$ for the eigenvalue 0 and $\mathrm{T} \mathbf{u}_{j}$ is an eigenvector of $S_{j}$ for the eigenvalue $-\frac{n}{2}$. 
Proof. The first statement is an immediate consequence of

$$
(A-t)^{-1}=C(\Lambda-t)^{-1} C^{-1}=\sum_{j=0}^{n} \frac{1}{\lambda_{j}-t} C E_{j} C^{-1}
$$

From $S_{j} C=-\mathrm{T} C E_{j}$ one sees that the vectors $\mathbf{u}_{i}$ with $i \neq j$ are eigenvectors of $S_{j}$ for the eigenvalue 0 and that $\mathrm{T} \mathbf{u}_{j}$ generates the image of $S_{j}$. So $\mathrm{T} \mathbf{u}_{j}$ is also an eigenvector of $S_{j}$ with eigenvalue equal to $\operatorname{trace}\left(S_{j}\right)=$ trace $\left(-C^{-1} \mathrm{~T} C E_{j}\right)$. The remaining eigenvalue thus equals the $(j, j)$ entry of the matrix $-C^{-1} \mathrm{~T} C$. Since $\left(C^{-1} \mathrm{~T} C\right)^{t}=C^{t} \mathrm{~T}\left(C^{t}\right)^{-1}=C^{-1} \mathrm{JTJ} C$, the diagonal of $C^{-1} \mathrm{~T} C$ equals $1 / 2$ times the diagonal of $C^{-1}(\mathrm{~T}+\mathrm{JTJ}) C=n \mathrm{l}$. Therefore, $\operatorname{trace}\left(S_{j}\right)=-n / 2$.

Theorem 6.3. Under the Assumption 6.1 the singularities of the system of differential equations

$$
\partial_{t} \Phi=\mathrm{T}(A-t)^{-1} \Phi
$$

are regular singular points located at $\infty$ and at the (distinct) eigenvalues $\lambda_{0}, \ldots, \lambda_{n}$ of $A$.

The monodromy transformation $M_{\infty}$ along a small positively oriented simple loop around $\infty$ is maximally unipotent, i.e., $\left(M_{\infty}-\mathrm{I}\right)^{n} \neq 0$, $\left(M_{\infty}-\mathrm{I}\right)^{n+1}=0$.

In case $n$ is odd the monodromy transformation $M_{j}$ along a small positively oriented simple loop around $\lambda_{j}$ has an eigenvalue 1 with $n$-dimensional eigenspace and an eigenvalue -1 with 1-dimensional eigenspace.

In case $n$ is even 1 is the only eigenvalue of the monodromy transformation $M_{j}$ along a small positively oriented simple loop around $\lambda_{j}$ and the dimension of the eigenspace is $\geqslant n$.

Proof. The proof of this theorem is given in the following section.

\subsection{Formal Fuchsian theory}

We borrowed the following concise account of it from [2, Chapter III, Section 8]. Let $G$ be a square matrix with entries in $\mathbb{C}[[x]]$. Write $D=x \frac{d}{d x}$. For an invertible matrix $H$ with entries in $\mathbb{C}\left[x, x^{-1}\right]$, define $G_{[H]}$ as $G_{[H]}=$ $(D H) H^{-1}+H G H^{-1}$. If a matrix $\Phi$ of functions in $x$ satisfies $D \Phi=G \Phi$, then $D(H \Phi)=G_{[H]}(H \Phi)$.

Let $\alpha$ be an eigenvalue of $G(0)$. Then according to [2, Lemma III. 8.2] there is an invertible matrix $H$ with entries in $\mathbb{C}\left[x, x^{-1}\right]$ such that the matrix 
$G_{[H]}$ has entries in $\mathbb{C}[[x]]$ and such that the matrix $G_{[H]}(0)$ has the same eigenvalues, counted with multiplicities, as $G(0)$, except that $\alpha$ is replaced by $\alpha+1$. A similar construction with $H^{-1}$ instead of $H$ replaces $\alpha$ by $\alpha-1$. By repeated application of this construction, we find an invertible matrix $H$ with entries in $\mathbb{C}\left[x, x^{-1}\right]$ such that the matrix $G_{[H]}(0)$ has prepared eigenvalues; i.e., the eigenvalues $\alpha_{0}, \ldots, \alpha_{n}$ satisfy: if $\alpha_{i} \in \mathbb{Z}$ then $\alpha_{i}=0$, and if $\alpha_{i}-\alpha_{j} \in \mathbb{Z}$ then $\alpha_{i}=\alpha_{j}$. The system $D \Psi=G_{[H]} \Psi$ then has a solution matrix of the form $\Psi=W \exp \left(G_{[H]}(0) \log x\right)$ where $W$ is a matrix with entries in $\mathbb{C}[[x]]$ such that $W(0)$ is the identity matrix. Thus, if $x$ runs through a small positively oriented loop about 0 the fundamental solution matrix $\Phi=H^{-1} \Psi$ gets multiplied from the right by $\exp \left(2 \pi i G_{[H]}(0)\right)$.

For the local monodromy of our system $\partial_{t} \Phi=\mathrm{T}(A-t)^{-1} \Phi$ around $\infty$ we use the local coordinate $x=t^{-1}$. This puts the system in the form

$$
x \frac{d}{d x} \Phi=G \Phi \quad \text { with } \quad G=\mathrm{T}(\mathrm{I}-A x)^{-1} .
$$

So, $G(0)=\mathrm{T}=\operatorname{diag}(0,1, \ldots, n)$. Take $H=\operatorname{diag}\left(1, x^{-1}, x^{-2}, \ldots, x^{-n}\right)$. Then

$$
G_{[H]}=(D H) H^{-1}+H G H^{-1}=\mathrm{T}\left(-\mathrm{I}+\sum_{k \geqslant 0}\left(x H A H^{-1}\right)^{k}\right) .
$$

We see that $G_{[H]}(0)$ is a nilpotent matrix with $G_{[H]}(0)^{n} \neq 0$ and $G_{[H]}(0)^{n+1}=0$. So, $G_{[H]}(0)$ has prepared eigenvalues and the monodromy is given by the maximally unipotent matrix $\exp \left(2 \pi i G_{[H]}(0)\right)$.

For the local monodromy around an eigenvalue $\lambda_{j}$ of $A$ we apply the general theory in the earlier paragraphs with $x=t-\lambda_{j}$ and $G=\mathrm{T} x(A-$ $\left.\lambda_{j}|-x|\right)^{-1}$. Then $G(0)=S_{j}$ as in Theorem 6.2.

Thus, if $n$ is odd $G(0)$ has prepared eigenvalues. The matrix for the monodromy around $\lambda_{j}$ is $\exp (2 \pi i G(0))$ and has an eigenvalue 1 with $n$-dimensional eigenspace and an eigenvalue -1 with 1-dimensional eigenspace.

If $n$ is even $G(0)$ does not have prepared eigenvalues. The general theory now provides a matrix $H$ such that 0 is the only eigenvalue of $G_{[H]}(0)$ and the eigenspace has dimension $\geqslant n$.

This completes the proof of Theorem 6.3.

Let us now turn to the local monodromies of the operator $L_{A, \infty}$ defined in 5.2. As we have seen in Section 4.4 the entries in the left-most column of the matrix $\Phi^{-1}$ (where $\Phi$ is as in Theorem 6.3) constitute a basis for the solution space of the differential equation $\operatorname{det}_{\text {right }}\left(t \partial_{t}-\tilde{A}\right) f=0$. So the local monodromies act on this basis by multiplication from the left by certain matrices $M_{\infty}^{-1}$ and $M_{j}^{-1}(j=0, \ldots, n)$. 
We know from Definition 5.2 that $\operatorname{det}_{\text {right }}\left(t \partial_{t}-\tilde{A}\right)=L_{A, \infty} \partial_{t}$. So the constant functions are solutions of the differential equation $\operatorname{det}_{\text {right }}\left(t \partial_{t}-\right.$ $\tilde{A}) f=0$ and the solution space of the differential equation $L_{A, \infty} g=0$ is obtained by taking the solution space of $\operatorname{det}_{\text {right }}\left(t \partial_{t}-\tilde{A}\right) f=0$ modulo the constant functions. Since the constant functions are fixed by all local monodromies we derive from Theorem 6.3 the following result about the monodromies of the operator $L_{A, \infty}$ :

Corollary 6.4. Under the Assumption 6.1 the differential equation

$$
L_{A, \infty} g=0
$$

has regular singularities located at $\infty$ and at the (distinct) eigenvalues $\lambda_{0}, \ldots, \lambda_{n}$ of $A$.

The local monodromy around $\infty$ is maximally unipotent, i.e., represented by an $n \times n$-matrix $\tilde{M}_{\infty}$ satisfying $\left(\tilde{M}_{\infty}-\mathrm{I}\right)^{n-1} \neq 0,\left(\tilde{M}_{\infty}-\mathrm{I}\right)^{n}=0$.

If $n$ is odd the local monodromy around $\lambda_{j}$ has eigenvalues 1 and -1 with eigenspaces of dimension $n-1$ and 1 , respectively.

If $n$ is even 1 is the only eigenvalue of the local monodromy around $\lambda_{j}$ and the dimension of the eigenspace is $\geqslant n-1$.

\section{Polarizability and generic irreducibility}

In this section, we prove that a generic operator of type DN is irreducible. The tactic is as follows. First, we prove that reducibility is a closed condition on an open set of the affine space of parameters of DNs. Up to this point we deal with the $\mathrm{DN}_{\infty, 1}$ flavor. Then we exhibit a single irreducible DN operator $\mathcal{H}$, for aesthetic reasons in the $\mathrm{DN}_{0,0}$ form, such that any operator in its neighborhood in the analytic topology is still irreducible.

We start, however, with the polarizability theorem:

Theorem 7.1. The monodromy of a differential operator $L$ of type $D N$ is polarized (i.e., its monodromy representation respects a non-degenerate bilinear form).

Proof. The operator $L$ determines a vector bundle over $\mathbb{A}^{1}(\mathbb{C}) \backslash\{$ singularities of $L\}$ and a connection in it. The adjoint gives rise to the dual connection in the dual bundle. Hence, the monodromy representation that corresponds to the adjoint is contragredient to the original one. We have proved in Theorem 4.6 that the differential operator $L$ of type $\mathrm{DN}_{\infty, 1}$ coincides, up to a sign, 
with its adjoint. Hence, its monodromy representation is isomorphic to its contragredient, and any such isomorphism corresponds to a non-degenerate bilinear form respected by the monodromy representation.

If a polarized monodromy representation is irreducible, then the polarizing bilinear form is defined uniquely up to a scalar (being an endomorphism of an irreducible object), and is either symmetric or skew depending on how the argument interchange involution acts on its span.

In our discussion of irreducibility, we consider differential operators with rational coefficients, i.e., elements of $\mathbb{C}(t)\left[\partial_{t}\right]$. As before, $N=n$.

Definition 7.2. Let $L=c_{n} \partial^{n}+c_{n-1} \partial^{n-1}+\cdots+c_{0} \in \mathbb{C}(t)[\partial]$, and $p$ be a point in $\mathbb{P}^{1}(\mathbb{C})$. The residue $\operatorname{res}_{p} L$ is by definition $\operatorname{res}_{p}\left(c_{n-1} / c_{n}\right) d t$.

Proposition 7.3. Let $L=c_{n} \partial^{n}+c_{n-1} \partial^{n-1}+\cdots+c_{0}$ be a differential operator of type $\mathrm{DN}_{\infty, 1}$ with $n+1$ distinct finite singularities. Then:

(i) The residue of $L$ at any finite singularity $p$ is $\frac{n}{2}$.

(ii) The residue of $L$ at infinity is $-n(n+1) / 2$.

Proof. Recall that we have identified L's of type $\mathrm{DN}_{\infty, 1}$ in Theorem 5.8(ii) as the differential operators of the form

$$
\left(t \partial_{t}\right)^{n} t+\sum_{p=1}^{n+1} g_{p}\left(t \partial_{t}\right) \partial_{t}^{p-1}
$$

with $g_{p}$ a polynomial of degree $\leqslant n-p+1$, that satisfies

$$
g_{p}(\operatorname{argument})=(-1)^{n-p+1} g_{p}(-\operatorname{argument}-p)
$$

Consider the $p$ th term to the right of the summation sign in the formula above. It can be presented as

$$
\bar{g}_{p}\left(t \partial_{t}+\frac{p}{2}\right) \partial_{t}^{p-1}
$$

where $\bar{g}_{p}$ is, depending on the parity of $n-p+1$, an even or odd polynomial (i.e., as a function of its argument). Let $\sum c_{i}^{(p)}(t) \partial_{t}{ }^{i}$ be the expansion of the $p$ th term.

To compare $c_{n}^{(p)}$ with $c_{n-1}^{(p)}$ it suffices to assume that $\bar{g}_{p}$ is a monomial (as a function of its argument) of the degree $n-p+1$ : the lower order terms 
contribute neither to $c_{n}^{(p)}$ nor to $c_{n-1}^{(p)}$. Inductively on $l$,

$$
\left(t \partial_{t}+\frac{p}{2}\right)^{l}=t^{l} \partial_{t}^{l}+\frac{l(l+p-1)}{2} t^{l-1} \partial_{t}^{l-1}+\cdots,
$$

and we compute $c_{n}^{(p)}=t^{n-p+1}$ and $c_{n-1}^{(p)}=\frac{(n-p+1) n}{2} t^{n-p}$, hence $c_{n-1}^{(p)}=\frac{n}{2} \frac{d c_{n}^{(p)}}{d t}$. Arguing in the same vein about $\left(t \partial_{t}\right)^{n} t=t\left(t \partial_{t}+1\right)^{n}$, we see, by linearity, that $c_{n-1}=\frac{n}{2} \frac{d c_{n}}{d t}$. Expanding $c_{n}$ into a series at a singularity proves the assertion (i).

Assertion (ii) follows because the sum of the residues of a rational function is 0 .

Definition 7.4. We say that a differential operator $L=c_{n} \partial^{n}+c_{n-1} \partial^{n-1}$ $+\cdots+c_{0} \in \mathbb{C}(t)[\partial]$ is irreducible if it cannot be represented as a product $L=L_{1} L_{2}$ with $L_{1}, L_{2} \in \mathbb{C}(t)[\partial]$ of positive order.

Consider the affine space $\mathbb{A}=\operatorname{Spec} \mathbb{C}\left[a_{i j}\right], 0 \leqslant i \leqslant j \leqslant n$, of differential operators of type DN.

Proposition 7.5. The locus of reducible DN operators is closed in a nonempty open subset of $\mathbb{A}$.

Proof. Let $\left\{L=c_{n} \partial_{t}{ }^{n}+c_{n-1} \partial_{t}{ }^{n-1}+\cdots+c_{0}\right\}$ be any set of differential operators with rational coefficients such that:

(i) the degrees of numerators and denominators of all $c_{i}$ are bounded.

(ii) there is a finite set $R \subset \mathbb{C}$ such that the residue at any singularity of any operator in the family is in $R$.

According to $[7$, section 9], there exists a positive integer $h$ such that for any factorization

$$
L=L^{\prime} L^{\prime \prime}
$$

where $L^{\prime}=\partial_{t}{ }^{n^{\prime}}+c_{n^{\prime}-1}^{\prime} \partial_{t}{ }^{n^{\prime}-1}+\cdots+c_{0}^{\prime}$ and $L^{\prime \prime}=c_{n^{\prime \prime}}^{\prime \prime} \partial_{t}^{n^{\prime \prime}}+c_{n^{\prime \prime}-1}^{\prime \prime} \partial_{t}{ }^{n^{\prime \prime}-1}$ $+\cdots+c_{0}^{\prime \prime}$ with $c_{i}^{\prime}, c_{i}^{\prime \prime} \in \mathbb{C}(t)$, the degrees of numerators and denominators of all $c_{i}^{\prime}, c_{i}^{\prime \prime}$ are bounded from above by $h$.

According to Proposition 7.3, the set D.S. of all DN's with $n+1$ distinct finite singularities satisfies hypotheses (i) and (ii) above. Therefore, the factorization (7.1) can be interpreted as defining a subscheme of the product of (an open subscheme of) the affine space $\mathbb{A}$ of the parameters $a_{i j}$ of DN and 
(open subschemes of) affine spaces of variable coefficients of the numerators and denominators of $c^{\prime}$ s and $c^{\prime \prime}$ s. By Chevalley's theorem ${ }^{2}$, the reducible locus is a finite union of locally closed subsets of D.S. Hence, reducibility is a closed condition on an open subset of $\mathbb{A}$.

Consider the operator

$$
\mathcal{H}=D^{n}-w^{n+1}(D+1)(D+2) \ldots(D+n) .
$$

Up to a scalar, it is the pullback of the regular hypergeometric operator

$$
\mathcal{H}^{\prime}=D_{u}^{n}-u\left(D_{u}+\frac{1}{n+1}\right)\left(D_{u}+\frac{2}{n+1}\right) \ldots\left(D_{u}+\frac{n}{n+1}\right)
$$

under the Kummer cover $u=w^{n+1}$. The coefficients of $\mathcal{H}^{\prime}$ at $u^{0}$ and $u^{1}$ are the polynomials $D_{u}^{n}$ and $\left(D_{u}+\frac{1}{n+1}\right)\left(D_{u}+\frac{2}{n+1}\right) \cdots\left(D_{u}+\frac{n}{n+1}\right)$ that have no common roots $\bmod \mathbb{Z}$. According to the classical criterion [8, 3.2.1], its monodromy is irreducible. It can be computed as in [1], or using the following proposition from [4]:

Proposition 7.6. $[4,1.2 .2]$. Let $F$ be a linear space endowed with a nondegenerate symmetric (or skew) form (, ). Let $U$ be a non-degenerate operator acting on $F$. Let $v$ be a cyclic vector for the operator $U$ and let $S$ be the reflection with respect to $v$ :

$$
S: x \mapsto x-(x, v) v
$$

Then

$$
1+\sum_{i=1}^{\infty}\left(U^{i} v, v\right) t^{i}=\frac{\operatorname{det}(1-t U S)}{\operatorname{det}(1-t U)}
$$

Consider the global monodromy of $\mathcal{H}^{\prime}$. Let $U$ be its monodromy around $\infty$ and $S$ be the monodromy around 1 . The monodromy around 0 is then the inverse of $U S$. Computing for instance by the standard Fuchsian procedure described in Section 6.1, one arrives at a classically known description of polarized hypergeometric monodromy [8, 3.2-3.3, 3.4, 3.5.4, 3.5.8]. There is a unique, up to a scalar, non-degenerate bilinear form respected by $U$ and $S$. It is symmetric for $n$ odd and skew for $n$ even. $S$ is a pseudoreflection ${ }^{3}$

\footnotetext{
${ }^{2}$ Under a morphism of finite type of Noetherian schemes, the image of a finite union of locally closed subsets is a finite union of locally closed subsets [6, Ex 3.19].

${ }^{3}$ I.e., $S-$ I has rank 1.
} 
in $O$ (resp. $S p$ ), hence a symmetric (resp. skew) reflection with respect to some vector $v$. US is unipotent. The eigenvalues of $U$ are all non-trivial $(n+1)$ th roots of unity, each with multiplicity 1 .

In particular, $v$ is a cyclic vector for $U$, because the $\mathbb{C}[U]$-span of $v$ is stable under $U$ and $S$ and because the monodromy representation of $\mathcal{H}^{\prime}$ is irreducible.

The global monodromy of $\mathcal{H}$ is the index $(n+1)$ subgroup of that of $\mathcal{H}^{\prime}$, generated, say, by the reflections with respect to $v, U v, U^{2} v, \ldots, U^{n} v$. Compute $\left(U^{j} v, U^{i}\right)$ by applying Proposition 7.6.:

$$
\left(U^{j} v, U^{i} v\right)=\left(U^{j-i} v, v\right)= \pm C_{n+1}^{j-i} \quad \text { for } 0 \leq i \neq j \leq n
$$

This shows that no two of $U^{i} v$ are orthogonal. Hence, the subgroup generated by these reflections acts on the fiber $F$ irreducibly.

Proposition 7.7. The operator $\mathcal{H}$ is of type $\mathrm{DN}_{0,0}$. It has $n+1$ distinct singularities and is irreducible.

Proof. The first assertion follows from the presentation in 5.8(iv), the second one is obvious. The third one was proved in the previous paragraph: in a non-trivial factorization $\mathcal{H}=\mathcal{H}_{1} \mathcal{H}_{2}$ both factors need to be regular singular, in particular, the local system of solutions of $\mathcal{H}_{2} \Phi=0$ would be a non-trivial subsystem of the one for $\mathcal{H}$.

Theorem 7.8. A generic differential operator $L$ of type $D N$ is irreducible.

Proof. We will spell out a semicontinuity argument in a sufficiently small open neighborhood of $\mathcal{H}$ in analytic topology of $\mathbb{A}(\mathbb{C})$ to show that the monodromy stays irreducible. By Corollory 6.4, the monodromy is generated by $n+1$ pseudoreflections of the form $x \mapsto x+h_{i}(x) v_{i}, i=0, \ldots, n$ where each $h_{i}$ stands for a non-zero covector in a fiber vector space, and each $v_{i}$, for a non-zero vector. Let $H_{i}=\operatorname{ker} h_{i}$.

Assume the monodromy representation is reducible, and $F_{0}$ is a monodromy-stable proper subspace of $F$. An alternative is now associated with each $v_{i}: F_{0}$ either contains $v_{i}$, or is in $H_{i}$. Reduce, if necessary, our neighborhood and note that $F_{0}$ cannot contain all $v$ 's (resp., be contained in all $H$ 's) because the subspace spanned by all $v$ 's is $F$ (resp., the intersection of all $H$ 's is zero), since this is the case for $\mathcal{H}$. Hence, there would exist $i$ and $j$ such that $v_{j} \in H_{i}$, which again is impossible because it does not happen for $\mathcal{H}$ : no bracket in formula 7.2 is zero. 
Summing up, the monodromy stays irreducible under deformation of $\mathcal{H}$ in the class of non-degenerate DNs; it implies that irreducibility is Zariski dense; we showed that reducibility is locally closed in Proposition 7.5. Therefore, the locus of irreducible DNs contains a non-empty Zariski open set.

A corollary is

Theorem 7.9. The monodromy representation of a generic (nondegenerate) operator $D N$ is polarizable by a bilinear form which is unique up to a scalar. The polarization is skew if $N$ is even and symmetric if $N$ is odd.

\section{Acknowledgment}

The authors thank NWO and RFBR (NWO-RFBR grant 047.017.019, NWO-RFBR grant 047.011.2004.026 [RFBR 05-02-89000-NWO_a] for Dutch Russian Research Cooperation) and the Mathematics Department of Utrecht University for financial support for visits, during which most research for this paper was done. V. Golyshev was supported by grant RUM1-2661-MO-05 of the U.S. Civilian Research and Development Foundation for the Independent States of the Former Soviet Union (CRDF). The authors are grateful to Victor Przyjalkowski for proofreading the note.

\section{References}

[1] F. Beukers, G. Heckman, Monodromy for the hypergeometric function ${ }_{n} F_{n-1}$. Invent. Math. 95 (1989), no. 2, 325-354.

[2] B. Dwork, G. Gerotto, F. Sullivan, An introduction to G-functions, Annals of Mathematics Studies, 133, Princeton University Press, Princeton, NJ, 1994.

[3] I. M. Gelfand, V. S. Retakh, Theory of noncommutative determinants, and characteristic functions of graphs, (Russian) Funktsional. Anal. i Prilozhen. 26 (1992), no. 4, 1-20; translation in Funct. Anal. Appl. 26 (1992), no. 4, 231-246 (1993).

[4] V. Golyshev, Riemann-Roch variations, Izv. akad. nauk, ser. mat. 65 (2001), no. 5, pp. 3-32.

[5] V. Golyshev, Classification problems and mirror duality, arXiv:math. AG/0510287. 
[6] R. Hartshorne, Algebraic geometry, Graduate Texts in Mathematics, 52, Springer-Verlag, New York-Heidelberg-Berlin, 1983.

[7] M. van Hoeij, Factorization of differential operators with rational functions coefficients. J. Symb. Comput. 24, (1997), no. 5, 537-561.

[8] N. Katz, Exponential sums and differential equations, Annals of Mathematics Studies, Princeton University Press, Princeton, NJ, 124, 1990.

[9] Yu. I. Manin, Frobenius Manifolds, Quantum cohomology and Moduli Spaces, AMS Colloquium Publications, 1999.

Number Theory SeCtion

Steklov Mathematical Institute

Russian ACADEMy of Sciences

8 GubKinA STR.

Moscow 119991

Russia

E-mail address: golyshev@mccme.ru

Department of Mathematics

UtRECHT UNIVERSITY

Postbus 80.010, 3508 TA

UTRECHT

The Netherlands

E-mail address: stien@math.uu.nl

Received February 7, 2007 